\title{
Frequency of Helicobacter pylori in patients hemodialysis its correlation with demographic and biochemical factors; a single center study
}

\author{
Ali Rostami, Hamid Nasri* ${ }^{\mathbb{D}}$ \\ Department of Internal Medicine, Isfahan University of Medical Science, Isfahan, Iran
}

Correspondence to:

Prof. Hamid Nasri, Email:

hamidnasri@yahoo.com,

hamidnasri@med.mui.ac.ir

Received: 5 Apr. 2021

Accepted: 19 June 2021

ePublished: 6 July 2021

Keywords: Helicobacter pylori, Hemodialysis, Biochemical factor, Demographic factor

\begin{abstract}
Introduction: Hemodialysis is one of the most common methods of treating chronic kidney disease (CKD). The start time of dialysis is determined depending on the clinical findings and subjective and objective parameters. However, factors such as patient perception and anxiety related to starting dialysis can modify these parameters. Patients on hemodialysis often have gastrointestinal complications; however, it is unclear whether Helicobacter pylori infection is more prevalent in these patients.

Objectives: The aim of this study was to investigate the relative frequency of levels of $\mathrm{H}$. pylori antibody in hemodialysis patients and its relationship with demographic and biochemical factors of hemodialysis patients in a hemodialysis center.

Patients and Methods: The present study is a cross-sectional and the target population of patients is the hemodialysis center of Amin hospital in 2019. The sampling method was a non-probabilistic sampling. The sample size in the present study was 85 people. The collected information was entered into SPSS software version 22 and analyzed with appropriate statistical tests. The significance level in the present study was less than 0.05 .

Results: In total, 85 patients participated in the present study, of which $68 \%$ were men and the rest were women. The cut-off point and the positive and negative border of laboratory results for $\mathrm{H}$. pylori were $18.8 \mathrm{U} / \mathrm{mL}$. The mean level of $H$. pylori antibody was $87.353 \pm 104.17 \mathrm{U} / \mathrm{mL}$. In this study, significant relationship between the level of $H$. pylori antibody and Parathyroid hormone (PTH) was detected. PTH level was lower in patients with a positive result for $H$. pylori. No further relationship was detected between H. pylori infection and biochemical parameters and blood indices, as well as $\mathrm{Kt} / \mathrm{V}$ and urea reduction rate (URR) was detected. Additionally, there was no significant difference in the mean level of $H$. pylori antibody between men and women. Accordingly, there was no significant correlation between H. pylori antibody level and age of patients, duration and number of dialysis sessions and body mass index.

Conclusion: Based on the findings of the present study, mean level of PTH was significantly lower in patients with a positive result for $H$. pylori. No significant relationship was observed between $H$. pylori infection with other demographic and biochemical factors.
\end{abstract}

\section{Introduction}

Chronic kidney disease (CKD) is defined as the structural or functional abnormality of the kidney with or without a reduction in glomerular filtration rate (GFR). People with CKD may have one or more of the following: Abnormalities, markers of kidney damage (ie, abnormal imaging and abnormal serum or urine including proteinuria and abnormal urinary sodium) or GFR less than $60 \mathrm{~mL} /$ min per 1.73 in square meter of body surface area for at least three months. If the duration of the abnormality is not known, the possibility of acute kidney damage should be considered and an appropriate evaluation performed for reversible causes. Most patients who have had a kidney transplant are considered to have CKD (1-3).

\section{Key point}

In a cross-sectional study on 85 hemodialysis patients, we found, no significant relationship between levels of Helicobacter pylori antibody with demographic factors.

Hemodialysis is one of the most common methods of treating CKD. The start time of dialysis is determined depending on the clinical findings and subjective and objective parameters. However, factors such as patient perception and anxiety related to starting dialysis can modify these parameters. Many guidelines recommend early initiation Dialysis has been performed in the presence of malnutrition and uremic syndrome, which can be lifesaving. The function of dialysis

Copyright ( 2021 The Author(s); Published by Society of Diabetic Nephropathy Prevention. This is an open-access article distributed under the terms of the Creative Commons Attribution License (http://creativecommons.org/licenses/by/4.0), which permits unrestricted use, distribution, and reproduction in any medium, provided the original work is properly cited. 
membranes includes the separation of large molecules and toxins. There are different types of dialysis membranes, each of which has different ability to separate molecules. Separate medium-sized ones as well (3-5).

Gastrointestinal complications in patients with renal insufficiency is one of the concerns and complaints of patients. Helicobacter pylori is one of the factors that play an important role in causing gastrointestinal complications, especially gastric ulcers $(6,7)$.

Complications of gastrointestinal bleeding are serious problems not only in patients with renal insufficiency but also in those undergoing hemodialysis. H. pylori is associated with cardiac complications as well as gastric malignancy in the long term, so its role is important in healthy individuals and hemodialysis patients (6-8).

\section{Objectives}

There are some debates about the association between H. pylori infection and CKD. Pathogenesis of H. pylori antibody in patients with CKD is not clearly revealed and there are not enough studies about these two factors. Therefore, the aim of this study was to investigate the relative frequency $H$. pylori antibody in hemodialysis patients and its relationship with demographic and biochemical factors of hemodialysis patients in hospitals in Isfahan.

\section{Patients and Methods}

\section{Study design}

The present study is a cross-sectional study and the target population of patients referred to the hemodialysis center of Amin hospital in 2018.

\section{Inclusion and exclusion criteria \\ Inclusion criteria}

Hemodialysis patients who referred to Amin hospital in 2018 with personal consent and have no severe systemic infection and infectious diseases and heart diseases including heart failure, coronary artery disease, respiratory distress, chronic obstructive pulmonary disease (COPD) and pregnancy.

\section{Exclusion criteria}

Dissatisfaction with the continuation of the study

\section{Sampling method and sample size}

The sampling method was a non-probabilistic sampling. The sample size in the present study was obtained using the following formula:

$$
\mathrm{n}=\frac{\left(Z_{1-\alpha / 2}+Z_{1-\beta}\right)^{2} s^{2}}{d^{2}}=85
$$

In this formula, based on a similar study, that $\mathrm{p}=0.4, \mathrm{~d}=$ $0.08, \alpha=0.05$ the sample size was 85 people.

\section{Data collection}

The method of data collection in the present study is as follows; people who referred to the hemodialysis section of Amin hospital in 2018 were asked about the age and underlying diseases and whether they were being treated for hypertension, diabetes and hyperlipidemia.

From the patients participating in the present study, height, weight and abdominal circumference indices were measured and recorded in their files and in individuals who meet the inclusion criteria, part of their blood serum is tested by isolated venous sampling. Then, the relationship between the data measured in the laboratory along with demographic data such as age, gender and duration of dialysis, the amount of fluid used in dialysis, which is collected by referring to the patient records in the hemodialysis ward of Amin hospital, along with Some biochemical indices such as dialysis fasting blood glucose and magnesium parathyroid hormone (PTH) and iron profile are collected by laboratory measurements.

\section{Ethical issues}

The research followed the tenets of the Declaration of Helsinki. The ethics committee of Isfahan University of Medical Sciences approved the ethical considerations of the present study (IR.MUI.MED.REC.1398.023). Accordingly, written informed consent was taken from all participants before any intervention. This article was extracted from the M.D, theses of Ali Rostami at this university (Thesis\# 397749).

\section{Statistical analysis}

The collected information was entered into SPSS software version 22 . The quantitative data were expressed as mean and standard deviation, and the qualitative data were assessed as numbers and percentages. KolmogorovSmirnov test was applied to analyze the normality of the data. Chi-square, $t$ test, Pearson's correlation coefficient and analysis of variance were performed to compare means of different. The significance level in the present study was less than 0.05 .

\section{Results}

In total, in the present study, 85 patients who underwent hemodialysis and referred to Amin hospital participated in the study. Descriptive and analytical results are presented below.

Out of a total of 85 patients, 58 patients (68\%) were male and the rest were female. The mean age of the patients was $62.80 \pm 13.76$ years. The mean body mass index (BMI) of patients was $24.25 \pm 4.9 \mathrm{~kg} / \mathrm{m}^{2}$. More than half of the patients were in the range of lean or normal weight. The mean dialysis dose was $10.87 \pm 2.22$ hours (18-4 hours). The cause of dialysis in half of patients was diabetes and the next cause was blood pressure. The access method for dialysis was arteriovenous fistula (AVF) in 39 patients and permcath in 46 patients.

Based on the results of the study, no significant relationship was observed between the level of $H$. pylori 
Table 1. Frequency distribution and percentage of background causes leading to dialysis in hemodialysis patients

\begin{tabular}{lcc}
\hline Background causes leading to dialysis & Number & Percent \\
\hline Diabetes & 40 & 0.147 \\
\hline Blood pressure & 24 & 28.2 \\
Diabetes and blood pressure (combined) & 5 & 5.9 \\
Systemic lupus erythematosus & 2 & 4.2 \\
Cholangiocarcinoma and cancer & 2 & 0.42 \\
\hline Glomerulonephritis and vasculitis & 2 & 4.2 \\
Other nephropathies (like IgA) & 2 & 4.2 \\
\hline Trauma & 1 & 1.2 \\
Proteinuria & 1 & 1.2 \\
Autosomal dominant polycystic kidney disease & 1 & 1.2 \\
Unknown & 5 & 5.9 \\
\hline Total & 85 & 100 \\
\hline
\end{tabular}

antibody and other biochemical parameters and blood indices, as well as $\mathrm{Kt} / \mathrm{V}$ and urea reduction rate (URR) and the level of $H$. pylori antibody and other parameters could not predict each other. Additionally, there was no significant difference in the mean level of $H$. pylori antibody between men and women.

Pearson's correlation coefficient indicates that there was no significant correlation between $H$. pylori antibody level and age of patients, duration and number of dialysis sessions and BMI. There was no significant difference in the mean level of $H$. pylori antibody in age groups.

Comparison of the mean level of $H$. pylori antibody in BMI groups did not show a significant difference. Comparison of $H$. pylori antibody levels in patients with different background causes of renal failure and dialysis did not show a significant difference.

Comparison of $H$. pylori antibody levels between patients with access to AVF and permcath for dialysis did not show a significant difference between these patients.

The distinguishing point in the results was the mean level of PTH, which was significantly different between patients with $H$. pylori antibody level of $18.8 \mathrm{U} / \mathrm{mL}$ and less on one side and more than $18.8 \mathrm{U} / \mathrm{mL}$, and was lower in patients with a positive result for $H$. pylori.

The mean level of $H$. pylori antibody was $87.353 \pm 104.17$ $\mathrm{U} / \mathrm{mL}$. The cut-off point and the positive and negative border of laboratory results for $H$. pylori were $18.8 \mathrm{U} /$ $\mathrm{mL}$ (according to the manufacturer's instructions for the kit) and based on this value, 30 patients were diagnosed negative with $H$. pylori infection and 55 patients were positive (Tables 1-6).

Table 2. Mean, standard deviation, minimum and maximum level of laboratory parameters in patients and its relationship with Helicobacter pylori antibody level

\begin{tabular}{|c|c|c|c|c|c|}
\hline Laboratory parameter & Mean & SD & Minimum & Maximum & Correlation with Helicobacter pylori antibody levels \\
\hline Helicobacter pylori antibody level (U/mL) & 87.353 & 104.17 & 1.3 & 0.357 & -- \\
\hline Calcium (mg/dL) & 322.8 & 62.0 & 6.5 & 10.3 & $r=-0.089, P=0.415$ \\
\hline Magnesium (mg/dL) & 2436.2 & 29.0 & 1.60 & 2.9 & $r=0.043, P=0.695$ \\
\hline Vitamin D (ng/ml) & 17.648 & 10.6 & 6.3 & 62.9 & $\mathrm{r}=0.079, P=0.474$ \\
\hline PTH (pg/mL) & 348.005 & 251.8 & 56.0 & 1818 & $r=-0.090, P=0.413$ \\
\hline Phosphorus (mg/dL) & 4.804 & 1.48 & 12 & 10.1 & $r=0.186, P=0.433$ \\
\hline Alkaline phosphatase (IU/L) & 401.359 & 281.49 & 2.5 & 1968 & $r=0.128, P=0.243$ \\
\hline Albumin $(\mathrm{g} / \mathrm{L})$ & 3.920 & 0.51 & 2.7 & 5.1 & $\mathrm{r}=0.143, P=0.191$ \\
\hline BUN (mg/dL) & 52.271 & 18.01 & 24.0 & 123 & $r=0.082, P=0.455$ \\
\hline Creatinine (mg/dL) & 7.119 & 2.56 & 2.2 & 16 & $\mathrm{r}=0.029, P=0.796$ \\
\hline $\mathrm{FBS}(\mathrm{mg} / \mathrm{dL})$ & 125.953 & 76.4 & 51.0 & 449 & $r=-0.168, P=0.124$ \\
\hline Cholesterol (mg/dL) & 133.965 & 36.85 & 70.0 & 242 & $r=-0.018, P=0.869$ \\
\hline Triglyceride (mg/dL) & 116.318 & 83.02 & 29.02 & 510 & $r=0.069, P=0.528$ \\
\hline Low-density lipoprotein cholesterol (mg/dL) & 72.800 & 24.05 & 26.0 & 146 & $r=0.021, P=0.849$ \\
\hline High-density lipoprotein cholesterol (mg/dL) & 33.659 & 11.59 & 15.0 & 76 & $r=-0.161, P=0.141$ \\
\hline White blood cell count $\left(\mathrm{mm}^{3}\right)$ & 6631.735 & 218.735 & 2000.0 & 12500 & $r=-0.116, P=0.292$ \\
\hline Red blood cell count $(6 * 10)\left(\mathrm{mm}^{3}\right)$ & 3.6629 & 0.66 & 1.59 & 5.06 & $r=0.050, P=0.653$ \\
\hline Platelet count $\left(\mathrm{mm}^{3}\right)$ & 183952.9 & 6380.638 & 31000.0 & 496000 & $\mathrm{r}=0.034, P=0.760$ \\
\hline Hemoglobin (g/dL) & 10.547 & 2.2 & 1.9 & 14.6 & $r=-0.131, P=0.233$ \\
\hline Hematocrit (\%) & 33.11 & 5.73 & 16.4 & 44.6 & $r=-0.103, P=0.346$ \\
\hline Serum iron $(\mathrm{mcg} / \mathrm{dL})$ & 115.9 & 182.48 & 8.0 & 947.8 & $r=-0.055, P=0.619$ \\
\hline Total iron binding capacity $(\mu \mathrm{g} / \mathrm{dL})$ & 284.28 & 107.46 & 3.8 & 829 & $r=-0.185, P=0.091$ \\
\hline Ferritin $(\mu \mathrm{g} / \mathrm{L})$ & 418.8 & 237.42 & 18.0 & 802 & $r=0.099, P=0.364$ \\
\hline $\mathrm{Kt} / \mathrm{V}^{*}$ & 1.249 & 0.35 & 0.46 & 2.1 & $r=-0.045, P=0.679$ \\
\hline URR & 0.64 & 0.11 & 0.32 & 0.98 & $r=-0.198, P=0.069$ \\
\hline
\end{tabular}

BUN, blood urea nitrogen; URR, urea reduction rate; FBS, fasting blood sugar.

* Kt/V: number used to quantify hemodialysis and peritoneal dialysis treatment adequacy. 


\section{Discussion}

Patients on hemodialysis often have gastrointestinal complications; however, it is unclear whether H. pylori infection is more than general population in these patients. Therefore, the aim of this study was to investigate the relative frequency of $H$. pylori antibody in hemodialysis patients and its relationship with demographic and biochemical factors of hemodialysis patients in a hemodialysis center in Isfahan.

In general, the results of the present study did not show a significant relationship between the prevalence of $H$. pylori

Table 3. Frequency distribution and percentage of positive and negative results of Helicobacter pylori antibody

\begin{tabular}{lcc}
\hline Helicobacter pylori & Number & Percent \\
\hline Positive (more than $18.8 \mathrm{U} / \mathrm{mL})$ & 55 & 64.7 \\
Negative $(18.8 \mathrm{U} / \mathrm{mL}$ and less) & 30 & 35.3 \\
Total & 85 & 100 \\
\hline
\end{tabular}

Table 4. Comparison of mean iPTH in patients with and without level of Helicobacter pylori antibody

\begin{tabular}{llccc}
\hline \multirow{2}{*}{$\begin{array}{l}\text { Helicobacter } \\
\text { pylori }\end{array}$} & Number & \multicolumn{2}{c}{ Level of PTH $(\mathbf{p g} / \mathbf{m L})$} & \multirow{2}{*}{$\boldsymbol{P}$ value } \\
\cline { 3 - 4 } & & Mean & SD & \\
\hline Positive & 58 & 297.78 & 322.6 & 0.012 \\
Negative & 27 & 440.06 & 108.20 & $\mathrm{t}=57.2$ \\
\hline
\end{tabular}

Table 5. Comparison of mean Helicobacter pylori antibody levels based on primary cause of chronic kidney disease

\begin{tabular}{lcc}
\hline Background cause & Number & $\begin{array}{c}\text { Helicobacter pylori } \\
\text { antibody }(\mathbf{U} / \mathbf{m L})\end{array}$ \\
\hline Diabetes & 40 & $81.73 \pm 106.4$ \\
\hline Blood pressure & 24 & $88.25 \pm 106.9$ \\
\hline Diabetes and blood pressure (combined) & 5 & $105.20 \pm 111.4$ \\
\hline Systemic lupus erythematosus & 2 & $53.50 \pm 41.7$ \\
Cholangiocarcinoma and cancer & 2 & $43.0 \pm 8.4$ \\
Glomerulonephritis and vasculitis & 2 & $126.50 \pm 164.7$ \\
Nephropathy & 2 & $289.5 \pm 0.7$ \\
\hline Trauma & 1 & 35.0 \\
\hline Proteinuria & 1 & 27.0 \\
\hline Autosomal dominant polycystic kidney & 1 & 153.0 \\
disease & 5 & $54.32 \pm 66.8$ \\
\hline Unknown & 85 & $87.35 \pm 104.1$ \\
\hline Total & &
\end{tabular}

$d f=10, P$ value $=0.439$.

Table 6. Comparison of mean Helicobacter pylori antibody levels based on hemodialysis accesses

\begin{tabular}{llccc}
\hline \multirow{2}{*}{ Dialysis access } & Number & \multicolumn{2}{c}{$\begin{array}{c}\text { Helicobacter pylori } \\
\text { antibody level }\end{array}$} & \multirow{2}{*}{$\boldsymbol{P}$ value } \\
\cline { 3 - 4 } & & Mean & SD & \\
\hline Arteriovenous fistula & 39 & 101.93 & 112.0 & 0.023 \\
Permcath & 46 & 74.99 & 96.4 & $\mathrm{t}=1.19$ \\
\hline
\end{tabular}

infection in hemodialysis patients and its relationship with demographic and biochemical factors of hemodialysis patients.

Other studies have reported conflicting results with the results of the present study. Based on other studies, the prevalence of $H$. pylori infection in patients receiving $\mathrm{CKD}$, chronic dialysis, or kidney transplant was reported to be equal or lower compared to subjects with normal renal function in various populations.

In a systematic review and meta-analysis study, Schein et al examined $H$. pylori infection in patients with CKD. The results of this study showed that, the total prevalence of $\mathrm{H}$. pylori infection was $48.2 \%$ in patients with CKD and $59.3 \%$ in the control group. Pooled analysis showed a significantly lower prevalence of $\mathrm{H}$. pylori infection in patients with CKD (versus control group: odds ratio, 0.64; 95\% confidence interval, 0.52 to 0.79 ) (9). The results of another meta-analysis study also showed that the rate of H. pylori infection in patients with CKD with and without dialysis was lower compared to patients without this disease (10).

The results of our study are consistent with some similar studies conducted in the past.

In a study, Najafi et al examined the prevalence of $H$. pylori in patients with chronic renal failure on dialysis. According to this study, chronic renal failure and dialysis do not change the prevalence of $H$. pylori infection. They also showed in this study that the prevalence of infection in all patients was $21.7 \%$ (15 patients). Around $80 \%$ of patients had gastrointestinal symptoms while about $21 \%$ of patients with gastrointestinal symptoms were positive for $H$. pylori (11).

A study in Japanese adults showed that the prevalence of $H$. pylori in patients with renal insufficiency was higher than the control group at about $70 \%$ (12). In subsequent studies, this difference was gradually reduced and similar to European and American reports, the prevalence of infection was reported to be the same in both groups with and without chronic renal failure (13).

According to current studies, the prevalence of infection in Japanese adults in dialysis and non-dialysis patients was not different. In other words, renal function has no significant effect on the prevalence of $H$. pylori (14).

In some studies, the prevalence of $H$. pylori infection in dialysis patients was lower than the control group. The study by Jaspersen et al, in adults showed that $H$. pylori was positive in $73.5 \%$ of adults with normal kidney function, while the prevalence was $26.6 \%$ in dialysis patients. Additionally, in the study of Yildiz et al, the prevalence of $H$. pylori infection was $72.5 \%$ in the control group and $65.9 \%$ in the dialysis group (15).

In nationwide study of Taiwan, $H$. pylori infection rate is lower in peptic ulcer disease patients with CKD and endstage renal disease (ESRD) than in those without CKD (16). On the contrary, recent meta-analysis studies showed 
no relationship between $H$. pylori infection and CKD or ESRD.

There are several hypotheses, which support that kidney disease may reduce $H$. pylori infection. High level of serum urea nitrogen can contribute to a decreased gastric acid secretion and higher gastric $\mathrm{pH}$, which could be the cause of lower prevalence of $H$. pylori among CKD $(17,18)$.

\section{Conclusion}

Based on the findings of the present study, mean level of PTH was significantly different between patients with $H$. pylori antibody level of $18.8 \mathrm{U} / \mathrm{mL}$ and less, and more than $18.8 \mathrm{U} / \mathrm{mL}$. Our study showed the lower PTH in patients with a positive result for $H$. pylori. No significant relationship was observed between $H$. pylori infection with other demographic and biochemical factors.

\section{Limitations of the study}

One of the limitations of the present study was the small sample size. It is suggested to use more sample size in other studies and also to use clinical trial and long-term or follow-up studies.

\section{Authors' contribution}

$H N$ and $A R$ were the principal investigators of the study. $H N$ and AR were included in preparing the concept and design. HN, AR revisited the manuscript and critically evaluated the intellectual contents. All authors participated in preparing the final draft of the manuscript, revised the manuscript and critically evaluated the intellectual contents. All authors have read and approved the content of the manuscript and confirmed the accuracy or integrity of any part of the work.

Conflicts of interest

The authors declare that they have no competing interests.

\section{Ethical consideration}

Ethical issues (including plagiarism, data fabrication, double publication, confidentiality of patients' information) have been completely observed by the authors.

\section{Funding/Support}

None.

\section{References}

1. Hakim RM, Lazarus JM. Initiation of dialysis. J Am Soc Nephrol. 1995;6:138.

2. Yang S, Xiao F, Pan L, Zhang H, Ma Z, Liu S, et al. Association of serum irisin and body composition with chronic kidney disease in obese Chinese adults: a cross-sectional study. BMC Nephrol. 2015;16:1

3. Sugano K, Tack J, Kuipers EJ, Graham DY, El-Omar EM, Miura $\mathrm{S}$, et al. Kyoto global consensus report on Helicobacter pylori gastritis. Gut. 2015;64:1353-67.
4. Ichikawa H, Sugimoto M, Sakao Y, Sahara S, Ohashi N, Kato $\mathrm{A}$, et al. Relationship between ghrelin, Helicobacter pylori and gastric mucosal atrophy in hemodialysis patients. World J Gastroenterol. 2016;22(47):10440-9. doi: 10.3748/wjg.v22. i47.10440

5. Bednarek-Skublewska A, Schabowski J, Majdan M, Baranowicz-Gaszczyk I, Ksiazek A. [Relationships between hyperparathyroidism and Helicobacter pylori infection in long-term hemodialysis patients]. Pol Arch Med Wewn. 2001;105:191-6

6. Ioannou GN, Weiss NS, Kearney DJ. Is Helicobacter pylori seropositivity related to body mass index in the United States? Aliment Pharmacol Ther. 2005;21:765-772.

7. Gu M, Xiao S, Pan X, Zhang G. Gastroenterol Res Pract. Helicobacter pylori Infection in Dialysis Patients: A MetaAnalysis. Gastroenterol Res Pract. 2013;2013:785892. doi: 10.1155/2013/785892.

8. Yang HR. Updates on the Diagnosis of Helicobacter pylori Infection in Children: What Are the Differences between Adults and Children? Pediatr Gastroenterol Hepatol Nutr. 2016;19:96-103. doi: 10.5223/pghn.2016.19.2.96

9. Shin SP, Bang CS, Lee JJ, Baik GH. Helicobacter pylori infection in patients with chronic kidney disease: a systematic review and meta-analysis. Gut Liver. 2019;13:628-641. doi: 10.5009/ gnl18517.

10. Ahn JY. Prevalence of Helicobacter pylori Infection in patients with chronic kidney disease. Gut Liver. 2019;13:585-6. doi: 10.5009/gnl19310.

11. Najafi M, Esfahani ST, Alahverdi B. Helicobacter pylori in patients with chronic renal failure. Iran J Pediatr. 2004;14:4752.

12. Asaka M, Kimura T, Kudo M, Takeda H, Mitani S, Miyazaki T, et al. Relationship of Helicobacter pylori to serum pepsinogens in an asymptomatic Japanese population. Gastroentrology. 1992;102:760-6

13. Gladziwa V, Haasc G, Handt S, Riehl J, Wietholtz HM Dakshinamurty KV, et al. Prevalence of Helicobacter pylori in patients with chronic renal failure. Nephrol Dial Transplant. 1993;8:301-6.

14. Jaspersen D, Fassbinder W, Heinkele P, Kronsbein H, Schorr W, Raschra C, et al. Significantly lower prevalence of Helicobacter pylori in uremic patients than in patients with normal renal function. J Gastroenterol. 1995;30:385 8.

15. 15-Yildiz A, Besisik F, Akkaya V, Sever MS, Bozfakioglu S, Yilmaz G, et al. Helicobacter pylori antibodies in hemodialysis patients and renal transplant recipients. Clin Tranplant. 1999;13:13-6.

16. 16.Chang SS, Hu HY. Lower Helicobacter pylori infection rate in chronic kidney disease and end stage renal disease patients with peptic ulcer disease. J Chin Med Assoc 2014;77:354-359.

17. 17.Wijarnpreecha K, Thongprayoon C, Nissaisorakarn P, Jaruvongvanich V, Nakkala K, Rajapakse R, et al. Association of Helicobacter pylori with chronic kidney diseases: a metaanalysis. Dig Dis Sci 2017;62:2045-2052.

18. 18.Wijarnpreecha K, Thongprayoon C, Nissaisorakarn P, Lekuthai N, Jaruvongvanich $\mathrm{V}$, Nakkala K, et al. Association between Helicobacter pylori and end-stage renal disease: a meta-analysis. World J Gastroenterol 2017;23:1497-1506. 